\title{
Systemic Immune-Inflammation Index (SII) Predicts Poor Survival in Pancreatic Cancer Patients Undergoing Resection
}

\author{
Gerd Jomrich $^{1}$ • Elisabeth S. Gruber ${ }^{1} \cdot$ Daniel Winkler $^{2} \cdot$ Marlene Hollenstein $^{1} \cdot$ Michael Gnant $^{1} \cdot$ Klaus Sahora $^{1}$. \\ Martin Schindl ${ }^{1}$
}

Received: 21 November 2018 / Accepted: 26 February 2019 / Published online: 28 March 2019

(C) 2019 The Author(s)

\begin{abstract}
Background The systemic immune-inflammation index based on peripheral neutrophil, lymphocyte, and platelet counts has shown a prognostic impact in several malignancies. The aim of this study was to determine the prognostic role of systemic immune-inflammation index in patients with pancreatic ductal adenocarcinoma undergoing resection.

Methods Consecutive patients who underwent surgical resection at the department of surgery at the Medical University of Vienna between 1995 and 2014 were included into this study. The systemic immune-inflammation index was calculated by the formula platelet*neutrophil/lymphocyte. Optimal cutoffs were determined using Youden's index. Uni- and multivariate analyses were calculated by the Cox proportional hazard regression model for overall survival.

Results Three hundred twenty-one patients were included in this study. Clinical data was achieved from a prospective patient database. In univariate survival analysis, elevated systemic immune-inflammation index was found to be significantly associated with shortened patients' overall survival $(p=0.007)$. In multivariate survival analysis, systemic immune-inflammation index remained an independent prognostic factor for overall survival $(p=0.004)$. No statistical significance could be found for platelet to lymphocyte ratio and neutrophil to lymphocyte ratio in multivariate analysis. Furthermore, area under the curve analysis showed a higher prognostic significance for systemic immune-inflammation index, compared to platelet to lymphocyte ratio and neutrophil to lymphocyte ratio.

Conclusion A high systemic immune-inflammation index is an independent, preoperative available prognostic factor in patients with resectable pancreatic ductal adenocarcinoma and is superior to platelet to lymphocyte ratio and neutrophil to lymphocyte ratio for predicting overall survival in pancreatic ductal adenocarcinoma patients.
\end{abstract}

Keywords Pancreatic ductal adenocarcinoma $\cdot$ Systemic immune-inflammation index $\cdot$ Inflammation

\section{Introduction}

Pancreatic ductal adenocarcinoma (PDAC) is the ninth most common malignancy and ranks fifth place of cancer-related death in western countries with inclining incidence. ${ }^{1}$ Despite the development of multimodal approaches, combining surgical resection with perioperative chemo-(radio)therapy, 5-year

Martin Schindl

martin.schindl@meduniwien.ac.at

1 Department of Surgery, Comprehensive Cancer Center (CCC), Medical University Vienna and Pancreatic Cancer Unit, Spitalgasse 23, 1090 Vienna, Austria

2 Vienna University of Economics and Business, Welthandelsplatz 1, AD 1020 Vienna, Austria survival rate for patients diagnosed with PADC remains poor with less than $5 \% .^{2}$

Inflammation, as one of the hallmarks of cancer, is an acknowledged factor in tumor biology. ${ }^{3,4}$ Inflammation-driven tumorigenesis and tumor progression plays a crucial role in malignant diseases. ${ }^{3,5}$ Systemic inflammatory response (SIR) in the context of tumor-associated inflammation has been demonstrated to diminish outcome and be of major prognostic importance in various cancers. ${ }^{6,7}$ A number of promising and potentially prognostic immunologic and histologic biomarkers have been investigated in PDAC. ${ }^{8,9}$ However, evaluation of these biomarkers is often expensive and time-consuming. Thus, investigation of tumor-driving inflammation-based components is of major importance and targeting pathways of inflammatory response might become a cornerstone of cancer treatment. ${ }^{10}$ In PDAC, outcome prediction mainly depends on 
clinical and pathological factors, such as tumor size, lymph node involvement, and distant metastases as well as resection margin. ${ }^{11}$ Notably, these factors are obtained post-operatively through evaluation of the surgical specimen and current available prognostic markers do not allow to preoperatively predict outcome. Therefore, identification of easy-available markers might help to determine individual treatment approaches. The utility of inflammation-based scores, such as neutrophillymphocyte ratio (NLR), platelet-lymphocyte ratio (PLR) and systemic immune-inflammation index (SII), are based on routinely obtained markers that are available before surgery. Elevated SII has been reported to be associated with clinico-pathological parameters and has been proven to be an independent prognostic factor in a number of malignancies, including PDAC. ${ }^{12-16}$ However, no data exists until now, describing the prognostic value of the SII in PDAC after neoadjuvant treatment. The aim of the present study was to assess the prognostic value of SII in patients undergoing a potentially curative resection for PDAC with or without neoadjuvant therapy. Furthermore, the SII is compared with NLR and PLR in predicting survival in this cohort of patients.

\section{Material and Methods}

Patients undergoing resection for PDAC between 1995 and 2014 at the Department of Surgery, Medical University of Vienna, were identified from a prospectively maintained PDAC database. Both patients who primarily underwent surgery and patients with borderline resectable disease according to NCCN guidelines who were treated by chemotherapy or radio-chemotherapy before resection were included. ${ }^{17}$ The study was approved by the Ethics Committee of the Medical University Vienna, Austria, in accordance with the Helsinki declaration (EK 1166/2013). Clinico-pathological data were assembled from medical records, including, gender, age, preoperative neutrophil, lymphocyte and platelet counts, tumor site, histopathological tumor grading, staging (TNM) according to the 8th edition of the Union for International Cancer Control (UICC)/American Joint Committee on Cancer (AJCC), neoadjuvant treatment and surgical resection technique. Tumor resection margin status $(R)$ was classified as $\mathrm{R} 0$ or R1 (1-mm tumor-free margin). Patients with distant metastases at time of diagnosis and death from other cause within 30 days post-surgery as well as patients who had recently pyrexia (axillary $\geq 37.2{ }^{\circ} \mathrm{C} / 99.0{ }^{\circ} \mathrm{F}$ ), any form of active infection or chronic inflammatory disease were excluded from the study. Each patient was discussed in the multidisciplinary team meeting before surgery. Neo-/adjuvant chemotherapy was administered according to the standard regimens available at the respective period. For neoadjuvant treatment, 5-fluorouracil was used from 1995 to 1998 , whereas gemcitabine-based regimens (gemcitabine monotherapy and combinations with oxaliplatin, erlotinib, and nab-Paclitaxel) or FOLFIRINOX were used from 1999 onwards. The present standard for neoadjuvant treatment since several years consists of either FOLFIRINOX or Gem/nab-Pac depending on patients' condition. For adjuvant chemotherapy, 5-fluorouracil-based regimens were used between 1995 and 1998 and gemcitabine-based regimens were administered thereafter. All patients were regularly followed thereafter with physical examination, tumor marker, and computed tomographic scan every 3 months for the first 2 years and every 6 months until 5 years after surgery.

Blood samples were obtained within 7 days prior to surgery. NLR, PLR, and SII were calculated as previously described: NLR = neutrophils / lymphocytes, PLR = platelet / lymphocytes, and SII = platelets $\times$ neutrophils / lymphocytes. ${ }^{16}$

\section{Statistical Analysis}

Statistical analysis was performed using the $\mathrm{R}$ statistical software (Version 3.4.3) with the "Survival," "pROC," and "Optimal Cutpoints" packages. ${ }^{18-21}$ To evaluate the discriminatory ability of the SII, NLR, and PLR, ROC curves were generated and the area under the ROC curves (AUROCs) was measured and compared. For bivariate analysis, to investigate relationships between SII, NLR, and PLR and clinico-pathological parameters, $t$ test and the Wilcoxon test were used as appropriate. Univariate and multivariate analyses were conducted using the Cox proportional hazard model. In the multivariable model, SII, NLR, and PLR could not be included into together, due to multicollinearity. Therefore, the stepwise regression analysis for multivariate Cox models, SII, NLR, and PLR could not be included into the model together due to multicollinearity. Discrimination ability was compared using the receiver operating curve. Optimal cutoff values for SII, NLR, and PLR were determined using Youden's index, which maximizes the sum of sensitivity and specificity. Graphically, it is represented by the distance between the 45-degree line and the ROC ${ }^{22,23}$. The graphical analysis was performed using the Kaplan-Meier survival curve estimator and analyzed by the log-rank test. Overall survival (OS) was defined as time between primary surgery and the patients' death. Death from other cause than PDAC or survival until the end of the observation period was considered as censored observations. Diseasefree survival (DFS) was defined from the day of surgery until first evidence of disease progression. Categorical data was analyzed using the chi-squared test. Continuous data was either analyzed using the $t$ test form normally distributed values or the Wilcoxon rank-sum test. 
Table 1 Association of the SII with clinico-pathological parameters in pancreatic ductal adenocarcinoma

\begin{tabular}{|c|c|c|c|c|c|}
\hline \multirow[t]{2}{*}{ Factors } & \multicolumn{2}{|l|}{ All } & \multicolumn{3}{|l|}{ SII } \\
\hline & & & High $(\geq 873)$ & Low $(<873)$ & $p$ value \\
\hline$n$ & 324 & & 120 & 204 & \\
\hline Age, mean (SD) & 68.25 & $(9.74)$ & $68.81(9.21)$ & $67.91(10.04)$ & $>0.1(*)$ \\
\hline Sex & & & & & $>0.1$ \\
\hline Male & 169 & $52.16 \%$ & 65 & 104 & \\
\hline Female & 155 & $47.84 \%$ & 55 & 100 & \\
\hline (y)pT & & & & & $>0.1$ \\
\hline 0 & 2 & $0.62 \%$ & 1 & 1 & \\
\hline 1 & 24 & $7.41 \%$ & 5 & 19 & \\
\hline 2 & 47 & $14.51 \%$ & 18 & 29 & \\
\hline 3 & 234 & $72.22 \%$ & 87 & 147 & \\
\hline 4 & 17 & $5.25 \%$ & 9 & 8 & \\
\hline (y)pN & & & & & $>0.1$ \\
\hline 0 & 86 & $26.54 \%$ & 27 & 59 & \\
\hline 1 & 238 & $73.46 \%$ & 93 & 145 & \\
\hline$(y) \mathrm{G}$ & & & & & $>0.1$ \\
\hline 1 & 15 & $4.63 \%$ & 4 & 11 & \\
\hline 2 & 204 & $62.96 \%$ & 72 & 132 & \\
\hline 3 & 105 & $32.41 \%$ & 44 & 61 & \\
\hline$R$ & & & & & 0.027 \\
\hline 0 & 254 & $78.40 \%$ & 86 & 168 & \\
\hline 1 & 70 & $21.60 \%$ & 34 & 36 & \\
\hline UICC staging & & & & & $>0.1$ \\
\hline I & 41 & $12.65 \%$ & 15 & 26 & \\
\hline II & 265 & $81.79 \%$ & 96 & 169 & \\
\hline III & 18 & $5.56 \%$ & 10 & 8 & \\
\hline Neoadjuvant treatment & & & & & $>0.1$ \\
\hline yes & 43 & $13.27 \%$ & 14 & 29 & \\
\hline no & 281 & $86.73 \%$ & 106 & 175 & \\
\hline Jaundice & & & & & 0.041 \\
\hline No & 163 & $50.31 \%$ & 51 & 112 & \\
\hline Yes & 161 & $49.69 \%$ & 69 & 92 & \\
\hline CA 19-9 & & & & & 0.013 \\
\hline Unknown & 24 & $7.41 \%$ & 9 & 15 & \\
\hline$\leq 114 \mathrm{kU} / \mathrm{L}$ & 143 & $44.14 \%$ & 42 & 101 & \\
\hline$>114 \mathrm{kU} / \mathrm{L}$ & 157 & $48.46 \%$ & 69 & 88 & \\
\hline Lymph node-ratio & & & & & $>0.1$ \\
\hline Unknown & 23 & $7.10 \%$ & 7 & 16 & \\
\hline$\leq 0.2$ & 196 & $60.49 \%$ & 71 & 125 & \\
\hline$>0.2$ & 105 & $32.41 \%$ & 42 & 63 & \\
\hline Nicotine & & & & & $>0.1$ \\
\hline Unknown & 3 & $0.93 \%$ & 1 & 2 & \\
\hline Yes & 135 & $41.67 \%$ & 55 & 80 & \\
\hline No & 186 & $57.41 \%$ & 64 & 122 & \\
\hline Pain & & & & & $>0.1$ \\
\hline Yes & 130 & $40.12 \%$ & 45 & 85 & \\
\hline No & 194 & $59.88 \%$ & 75 & 119 & \\
\hline Pancreatitis & & & & & $>0.1$ \\
\hline Yes & 63 & $19.44 \%$ & 20 & 43 & \\
\hline No & 261 & $80.56 \%$ & 100 & 161 & \\
\hline Diabetes & & & & & $>0.1$ \\
\hline Unknown & 12 & $3.70 \%$ & 3 & 9 & \\
\hline Yes & 73 & $22.53 \%$ & 29 & 44 & \\
\hline No & 239 & $73.77 \%$ & 88 & 151 & \\
\hline Stent & & & & & $>0.1$ \\
\hline Yes & 153 & $47.22 \%$ & 60 & 93 & \\
\hline No & 171 & $52.78 \%$ & 60 & 111 & \\
\hline Surgical procedure & & & & & 0.0345 \\
\hline PPPD & 181 & $55.86 \%$ & 65 & 116 & \\
\hline Whipple & 71 & $21.91 \%$ & 35 & 36 & \\
\hline Distal resection & 60 & $18.52 \%$ & 15 & 45 & \\
\hline Total pancreatectomy & 12 & $3.70 \%$ & 5 & 7 & \\
\hline Localization & & & & & $>0.1$ \\
\hline 1 & 255 & $78.70 \%$ & 100 & 155 & \\
\hline 2 & 24 & $7.41 \%$ & 8 & 16 & \\
\hline 3 & 45 & $13.89 \%$ & 12 & 33 & \\
\hline
\end{tabular}

(*) Using $t$ test

SD, standard deviation; UICC, Union for International Cancer Control; SII, systemic immune-inflammation index 


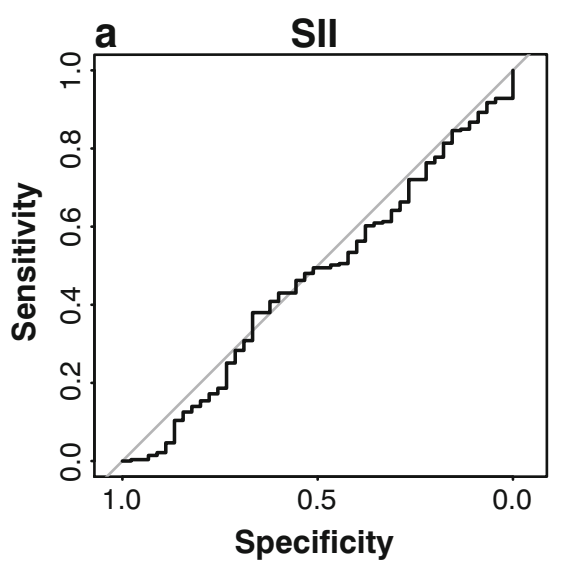

Fig. 1 Receiver operating characteristic (ROC) curves were generated to evaluate the discriminatory ability of the SII (a), NLR (b), and PLR (c).

\section{Results}

In the present study, a total of 321 patients $(169,52.2 \%$ male $)$ with a median age of 68.5 (range, 35.9-92.3) years were included. The majority of patients $(201,62.6 \%)$ presented with a moderately differentiated tumor (G2) and 43 (13.3\%) patients received neoadjuvant treatment prior to resection. In 255 (78.7\%) cases, the tumor was located in the head of the pancreas and in $238(73.5 \%)$ positive lymph node was found at pathological assessment. In accordance with the 8th edition UICC/AJCC classification, 265 (82.6\%) patients showed stage II disease and 153 (47.2\%) patients underwent biliary drainage preoperatively. Clinico-pathological data are presented in Table 1.

The median OS was 18.5 months (range, 1.5198 months) and the rate of 3- and 5-year OS was $25.63 \%$ and $8.44 \%$, respectively. The optimal cutoff values for SII, PLR, and NLR were 873, 179, and 225, respectively. With the defined cutoffs, 119 patients had SII $\geq 873,125$ patients had PLR $\geq 179$, and 225 patients NLR $\geq 2.15$ before surgery. Using bivariate analysis, significant relationship between elevated SII and clinicopathological parameters was found for resection margin $(p=0.03)$, jaundice $(p=0.04)$, CA 19-9 $(p=0.01)$, and surgical procedure $(p=0.04)$ (Table 1$)$. The median OS
NLR

Shes

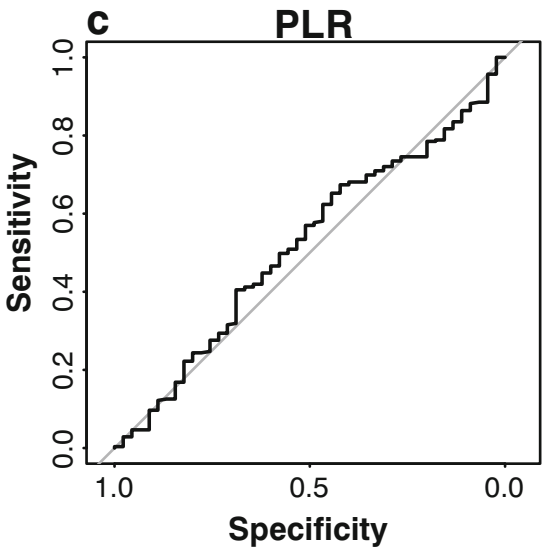

The area under the ROC curves (AUROCs) were measured and compared

for patients with high SII was 14.2 (range, 1.5-128.2) months and 20.5 (range, 1.6-200.8) months for patients with low SII respectively. In the entire cohort, using overall survival as an end-point, the area under the receiver operator curve was $0.46(\mathrm{CI}, 0.37-0.55)$ for SII, 0.46 (CI, 0.36-0.56) for NLR, and 0.51 (CI, 0.42-0.61) for PLR with no significant difference in discrimination ability between SII, NLR, and PLR regarding OS was found (Fig. 1).

Kaplan-Meier curve survival analysis for all patients revealed that low SII $(p=0.004)$ and PLR $(p=0.04)$ were significantly associated with longer OS, whereas no significance was found for NLR (Fig. 2).

Univariate Cox proportional hazard regression revealed that SII, PLR, age, jaundice, resection margin, CA 19-9, lymph node-ratio, and tumor size were significantly associated with OS. Similarly, age, jaundice, lymph node-ratio, and tumor size are significantly associated with DFS whereas SII, NLR, and PLR was not (Table 2).

Furthermore, no statistical significance was found for OS and DFS in univariate Cox proportional hazard regression for neoadjuvant treatment $(p=0.08 ; \mathrm{RR}, 1.35 ; \mathrm{CI}, 950.96-1.88$; and $p=$ 0.23; RR, 1.24; CI, 95 0.87-1.77; respectively; Table 2).

Multivariate Cox-regression analysis using SII as bivariate variable revealed that a high SII $(p=0.016$; RR,
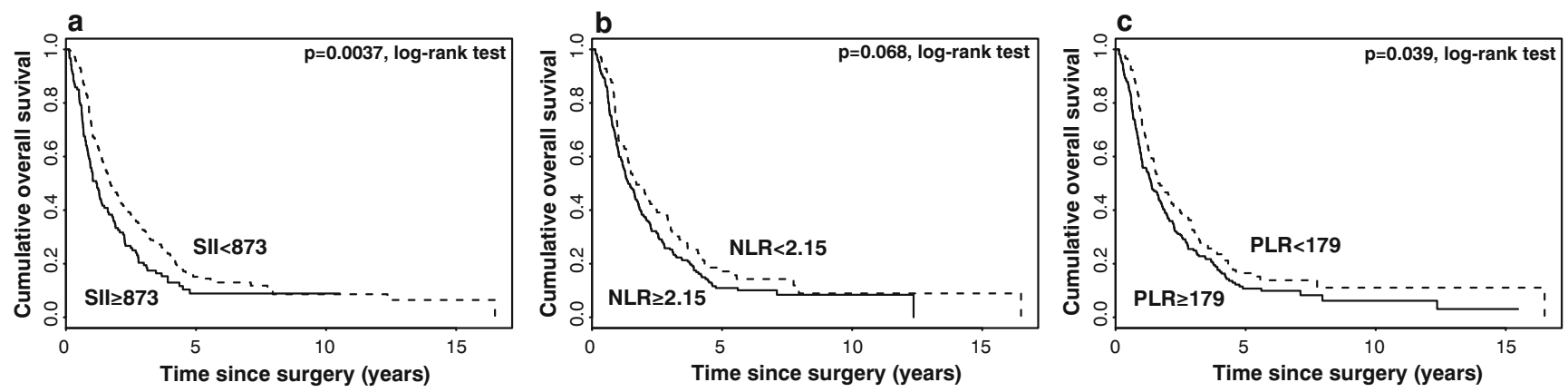

Fig. 2 Kaplan-Meier curves for overall survival (OS) stratified by SII (a), NLR (b) and PLR (c) 
Table 2 Univariate Cox regression analysis estimating the influence of the SII, NLR, PLR and clinico-pathological parameters on overall survival and disease-free survival in patients with ductal adenocarcinoma of the pancreas

\begin{tabular}{|c|c|c|c|c|c|c|}
\hline Overall survival & $\mathrm{RR}$ & CI $(95 \%)$ & $p$ value & $\begin{array}{l}\text { Disease-free survival } \\
\text { RR }\end{array}$ & CI $(95 \%)$ & $p$ value \\
\hline \multicolumn{7}{|l|}{ SII } \\
\hline$\geq 873$ vs. $<873$ & 0.6979 & $0.5469-0.8907$ & 0.0038 & 0.8147 & $0.6288-1.0556$ & 0.1211 \\
\hline \multicolumn{7}{|l|}{ NLR } \\
\hline \multicolumn{7}{|l|}{ PLR } \\
\hline $\begin{array}{l}\geq 179 \text { vs. }<179 \\
\text { UICC stage }\end{array}$ & \multicolumn{2}{|c|}{ UICC stage } & & 0.808 & $0.6205-1.0522$ & 0.1136 \\
\hline II vs. I & 1.0668 & $0.7438-1.5301$ & 0.7252 & 1.1808 & $0.7898-1.7653$ & 0.4179 \\
\hline III vs. I & 1.4071 & $0.7658-2.5852$ & 0.2712 & 1.2352 & $0.6366-2.3964$ & 0.5323 \\
\hline \multicolumn{7}{|l|}{ Grading } \\
\hline 2 vs. 1 & 0.7087 & $0.4174-1.2032$ & 0.2023 & 0.6882 & $0.3973-1.1922$ & 0.1826 \\
\hline 3 vs. 1 & 1.0347 & $0.5996-1.7855$ & 0.9026 & 1.0086 & $0.5719-1.7785$ & 0.9765 \\
\hline \multicolumn{7}{|l|}{ Sex } \\
\hline Male vs. female & 1.0843 & $0.8562-1.373$ & 0.5019 & 1.0475 & $0.8145-1.3472$ & 0.7175 \\
\hline Age & 0.9881 & $0.9769-0.9993$ & 0.038 & 0.9838 & $0.9714-0.9964$ & 0.0116 \\
\hline \multicolumn{7}{|l|}{ Jaundice } \\
\hline Yes vs. no & 1.4243 & $1.1246-1.8039$ & 0.0033 & 1.4186 & $1.1013-1.8272$ & 0.0068 \\
\hline \multicolumn{7}{|l|}{$R$} \\
\hline 1 vs. 0 & 1.4382 & $1.0866-1.9037$ & 0.0111 & 1.3526 & $0.9989-1.8315$ & 0.0508 \\
\hline \multicolumn{7}{|l|}{ CA $19-9$} \\
\hline$\geq 114$ vs. $<114 \mathrm{kU} / \mathrm{L}$ & 1.3148 & $1.0281-1.6815$ & 0.0292 & 1.2842 & $0.9899-1.6661$ & 0.0596 \\
\hline \multicolumn{7}{|l|}{ Lymph node-ratio } \\
\hline$\geq 0.2$ vs. $<0.2$ & 1.8094 & $1.4006-2.3375$ & $<0.0001$ & 1.6615 & $1.264-2.184$ & 0.0003 \\
\hline \multicolumn{7}{|l|}{ T-staging } \\
\hline 1 vs. 0 & 0.2554 & $0.0592-1.1006$ & 0.067 & 0.1249 & $0.0162-0.9622$ & 0.0458 \\
\hline 2 vs. 0 & 0.231 & $0.0553-0.9647$ & 0.0445 & 0.1209 & $0.0162-0.9034$ & 0.0395 \\
\hline 3 vs. 0 & 0.2674 & $0.0657-1.0876$ & 0.0654 & 0.1266 & $0.0173-0.9263$ & 0.0418 \\
\hline 4 vs. 0 & 0.3471 & $0.0786-1.5319$ & 0.1624 & 0.1349 & $0.0172-1.0561$ & 0.0564 \\
\hline \multicolumn{7}{|l|}{$\mathrm{N}$-staging } \\
\hline 1 vs. 0 & 1.158 & $0.8836-1.5176$ & 0.2876 & 1.0838 & $0.8162-1.4391$ & 0.5781 \\
\hline \multicolumn{7}{|l|}{ Pain } \\
\hline Yes vs. no & 0.9929 & $0.7804-1.2632$ & 0.9535 & 1.0445 & $0.8088-1.3488$ & 0.7388 \\
\hline \multicolumn{7}{|l|}{ Pancreatitis } \\
\hline Yes vs. no & 0.9514 & $0.7064-1.2815$ & 0.7431 & 0.8858 & $0.6426-1.2212$ & 0.4592 \\
\hline \multicolumn{7}{|l|}{ Nicotine } \\
\hline Yes vs. no & 1.106 & $0.8705-1.406$ & 0.409 & 1.0901 & $0.8457-1.4052$ & 0.5053 \\
\hline \multicolumn{7}{|l|}{ Diabetes } \\
\hline Yes vs. no & 1.136 & $0.8545-1.51$ & 0.38 & 1.1107 & $0.8214-1.502$ & 0.4953 \\
\hline \multicolumn{7}{|l|}{ Neoadjuvant treatment } \\
\hline Yes vs. no & 1.3449 & $0.9616-1.881$ & 0.0834 & 1.2416 & $0.8711-1.7696$ & 0.2314 \\
\hline \multicolumn{7}{|l|}{ Stent } \\
\hline Yes vs. no & 1.1175 & $0.883-1.4142$ & 0.3553 & 1.1664 & $0.9076-1.4989$ & 0.2291 \\
\hline \multicolumn{7}{|l|}{ Surgical procedure } \\
\hline \multicolumn{7}{|l|}{ PPPD } \\
\hline Whipple & 1.0712 & $0.7961-1.4414$ & 0.6497 & 1.0691 & $0.7808-1.4638$ & 0.6769 \\
\hline Distal resection & 0.8498 & $0.6152-1.1739$ & 0.3236 & 1.2939 & $0.6334-2.6432$ & 0.4796 \\
\hline Total pancreatectomy & 1.4092 & $0.7425-2.6747$ & 0.294 & 0.9609 & $0.6824-1.353$ & 0.8192 \\
\hline \multicolumn{7}{|l|}{ Localization } \\
\hline 2 vs. 1 & 0.8828 & $0.5386-1.4469$ & 0.621 & 1.0087 & $0.6055-1.6802$ & 0.9736 \\
\hline 3 vs. 1 & 0.9289 & $0.6605-1.3062$ & 0.6714 & 1.0415 & $0.7199-1.5069$ & 0.829 \\
\hline
\end{tabular}

UICC, Union for International Cancer Control; CI, confidence interval; RR, relative risk; Ref., reference; SII, systemic immune-inflammation index; PLR, platelet lymphocyte ratio; NLR, neutrophil lymphocyte ratio; PPPD, pylorus-preserving pancreaticoduodenectomy

0.71 ; CI, 95\% 0.54-0.94), positive resection margin ( $p=$ 0.03; RR, 1.46; CI, 95\% 1.05-2.03), and a high lymph node-ratio $(p<0.001$; RR, 1.77; CI, 95\% 1.32-2.36), but not NLR and PLR, are independent risk factors for OS (Table 3). No statistical significant association could be found for SII, NLR, and PLR in the multivariate Cox models for DSF (Table 4).

\section{Discussion}

Inflammation plays a key role in tumor initiation, malignant conversion, and metastasis and influences the host anti-tumor immunity. ${ }^{3-5}$ The present study investigated the clinical and prognostic value of preoperative SII, NLR, and PLR in patients with PDAC undergoing resection and competed their 
Table 3 Multivariate Cox regression analysis estimating the influence of the SII and clinico-pathological parameters on overall survival in patients with ductal adenocarcinoma of the pancreas

\begin{tabular}{|c|c|c|c|}
\hline Overall survival & $\begin{array}{l}\text { SII } \\
\text { RR }\end{array}$ & CI $(95 \%)$ & $p$ value \\
\hline \multicolumn{4}{|l|}{ SII } \\
\hline$\geq 873$ vs. $<873$ & 0.7138 & $0.5427-0.9388$ & 0.0159 \\
\hline \multicolumn{4}{|l|}{ UICC stage } \\
\hline II vs. I & 0.7796 & $0.493-1.2329$ & 0.2871 \\
\hline III vs. I & 0.9423 & $0.4591-1.9344$ & 0.8714 \\
\hline \multicolumn{4}{|l|}{ Grading } \\
\hline 2 vs. 1 & 0.5881 & $0.3181-1.0875$ & 0.0906 \\
\hline 3 vs. 1 & 0.8104 & $0.4285-1.5328$ & 0.5179 \\
\hline \multicolumn{4}{|l|}{ Sex } \\
\hline Male vs. female & 0.9328 & $0.7155-1.2162$ & 0.6074 \\
\hline Age & 0.9874 & $0.9749-1.0001$ & 0.0514 \\
\hline \multicolumn{4}{|l|}{ Jaundice } \\
\hline Yes vs. no & 1.1994 & $0.9094-1.5819$ & 0.1979 \\
\hline \multicolumn{4}{|l|}{$R$} \\
\hline 1 vs. 0 & 1.4561 & $1.0457-2.0275$ & 0.0261 \\
\hline \multicolumn{4}{|l|}{ CA 19-9 } \\
\hline$\geq 114$ vs. $<114 \mathrm{kU} / \mathrm{L}$ & 1.1642 & $0.8795-1.541$ & 0.288 \\
\hline \multicolumn{4}{|l|}{ Lymph node-ratio } \\
\hline$\geq 0.2$ vs. $<0.2$ & 1.7687 & $1.3247-2.3615$ & $<0.001$ \\
\hline
\end{tabular}

UICC, Union for International Cancer Control; CI, confidence interval; $\mathrm{RR}$, relative risk; SII, systemic immune-inflammation index

predictive accuracy. Overall, SII, but not NLR and PLR was an independent prognostic factor for OS in patients with PDAC undergoing resection.

The relationship between tumor and inflammation was first described by Virchow in 1863, and later in 1986 by Dvorak as "Tumors: Wounds that do not heal." Meanwhile, inflammation is known as one of the hallmarks of cancer. ${ }^{4,24,25}$ Increasing data shows the close relationship between tumorigenesis, tumor progression, and metastasis. ${ }^{4-6}$ The major prognostic impact of inflammatory markers can be ascribed to a cytokine-driven immunogenic tumor microenvironment and a significant prognostic role of inflammation-based biomarkers and scores has recently been shown in a number of malignant diseases. ${ }^{5,7,26-28}$ One of the newly emerging prognostic scores is the SII, based on platelets, neutrophils, and lymphocytes. As a combination of both NLR and PLR, SII firstly has been confirmed as superior prognostic factor in hepatocellular carcinoma and then in small cell lung cancer reflecting patient's inflammatory status. ${ }^{15,16}$ In a number of malignancies, including PDAC, an elevated preoperative SII plays a key role in prognosis estimation. ${ }^{12-16,29-31}$ This is the first study that has proven the prognostic value of the SII and is superior to PLR.

It has been proposed that SII is able to predict tumor recurrence in a highly inflammatory tumor microenvironment with
Table 4 Multivariate Cox regression analysis estimating the influence of the SII and clinico-pathological parameters on disease-free survival in patients with ductal adenocarcinoma of the pancreas

\begin{tabular}{|c|c|c|c|}
\hline Disease-free survival & $\begin{array}{l}\text { SII } \\
\text { RR }\end{array}$ & CI $(95 \%)$ & $p$ value \\
\hline \multicolumn{4}{|l|}{ SII } \\
\hline$\geq 873$ vs. $<873$ & 0.787 & $0.5885-1.0524$ & 0.1062 \\
\hline \multicolumn{4}{|l|}{ UICC stage } \\
\hline II vs. I & 1.0855 & $0.6388-1.8446$ & 0.7617 \\
\hline III vs. I & 1.0861 & $0.495-2.3831$ & 0.8367 \\
\hline \multicolumn{4}{|l|}{ Grading } \\
\hline 2 vs. 1 & 0.5279 & $0.2826-0.9861$ & 0.0451 \\
\hline 3 vs. 1 & 0.7407 & $0.3878-1.4148$ & 0.3633 \\
\hline \multicolumn{4}{|l|}{ Sex } \\
\hline Male vs. female & 0.9176 & $0.6892-1.2217$ & 0.5562 \\
\hline Age & 0.9823 & $0.9685-0.9964$ & 0.0138 \\
\hline \multicolumn{4}{|l|}{ Jaundice } \\
\hline Yes vs. no & 1.2138 & $0.9041-1.6295$ & 0.1973 \\
\hline \multicolumn{4}{|l|}{$R$} \\
\hline 1 vs. 0 & 1.405 & $0.984-2.006$ & 0.0613 \\
\hline \multicolumn{4}{|l|}{ CA 19-9 } \\
\hline$\geq 114$ vs. $<114 \mathrm{kU} / \mathrm{L}$ & 1.1385 & $0.8459-1.5323$ & 0.3921 \\
\hline \multicolumn{4}{|l|}{ Lymph node-ratio } \\
\hline$\geq 0.2$ vs. $<0.2$ & 1.4942 & $1.0995-2.0306$ & 0.0103 \\
\hline
\end{tabular}

UICC, Union for International Cancer Control; CI, confidence interval; $\mathrm{RR}$, relative risk; SII, systemic immune-inflammation index

infiltrating immune cells that promote tumorigenesis and dissemination. ${ }^{5,32}$ Neutrophils activate endothelium and parenchymal cells via secretion of soluble factors that enhance tumor cell adhesion at distant sites. ${ }^{33-35}$ Increasing numbers of blood neutrophils and platelets have been associated with tumor progression and diminished clinical outcome in a number of solid tumors. ${ }^{36,37}$ Lymphocytes inhibit tumor cell proliferation and migration through induction of cytotoxic cell death and thus play a key role in cancer immuno-surveillance. ${ }^{5}$ On the basis of these findings, several inflammation-based scores have emerged as prognostic indicators in cancer patients.

Recently published data is diverging regarding the prognostic value of NLR and PLR. The NLR, combining circulating neutrophil and lymphocyte counts, and the PLR, combining circulating platelets and lymphocyte counts, has been associated with impaired survival in lung and ovarian cancers, while in PDAC results remain inconsistent. ${ }^{38-42}$ Whereas Mowbray et al. found preoperative NLR to be an independent prognostic predictor, Chawla et al. reports that neither NLR nor PLR predicts survival in patients who underwent pancreatectomy for PDAC. ${ }^{43,44}$

A high SII, consisting of high neutrophil and platelet as well as low lymphocyte counts, indicates inflammation activity that may be associated with poor survival through enhanced tumor invasion and metastases. Investigating 
the prognostic capacity of SII, NLR, and PLR, our results were consistent with those of Chawla et al., revealing that preoperative SII, but not NLR and PLR, is an independent prognostic factor for OS in patients with resectable PDAC. ${ }^{43}$ Recently, Haldar and Ben-Eliyahu critically discussed the impact of perioperative $\beta$-adrenergic blockade and COX2 inhibition on cancer outcomes. ${ }^{45}$ Thus, patients with resectable PDAC who have elevated preoperative SII might benefit from anti-inflammatory and/or antiimmunotherapy prior and after surgery.

Even though the results of the present study demonstrate that the SII is an independent prognostic factor in patients with PDAC undergoing resection, it has several limitations. Although the patients were prospectively entered into a database, a retrospective analysis was performed with a selection bias by the availability of complete blood cell count before surgery in daily practice. The cohort represents the experience of one center that needs to be validated by external cohort from another center.

There are no consensual cutoff values for inflammation indices. The majority of studies determine individual cutoff levels by their relevance and significance, showing a significant prediction of survival when applied to the same patients' cohort. As a result, there is a wide range of cutoff values for these indices. However, the present study demonstrated that the SII provided the strongest survival prediction compared to NLR and PLR in patients with PDAC undergoing surgery. Emphasis should be given to determine significant cutoff levels for inflammatory indices that stay valid when applied to independent cohorts of patients.

The administration of different neo-/adjuvant chemotherapy regimens and changing policies of treating patients with borderline disease during the study period may have influenced the study result. However, it reflects the real-world situation and patients included in the study were treated with standard regimens that were available at the respective period. We did not analyze the differences of prognostic strength of SII during different time intervals reflecting variations in neo-/ adjuvant chemotherapy regimens. However, we could proof the prognostic value of SII for the entire observation period, regardless neoadjuvant treatment was administered or not. We admit that in order to draw representative conclusions for the association between inflammatory activity and prognosis in patients undergoing neoadjuvant treatment, a cohort with little variations in neoadjuvant regimens should be analyzed.

High and low SII was equally distributed in most of the items characterizing the study cohort. However, resection rates, CA19-9 and bilirubin blood concentrations were different between patients with high and low inflammatory activity. The difference of resection rates interesting observation that needs to be addressed by further studies as it may represent an important factor for treatment decision. There is evidence that tumor invasion initiates host inflammatory response and one could argue that the extent of tumor cell infiltration into the mesopancreatic compartment both stimulates inflammatory activity and influences the likelihood of complete resection. Similarly, CA19-9, serves as a surrogate marker of tumor burden and thereby associated with inflammation.

\section{Conclusion}

In summary, the present study shows that preoperative SII is an independent predictor of OS in patients with PDAC undergoing pancreatic resection that is superior to NLR and PLR. Measurement of SII is easily applicable and of low cost. Patients with preoperatively elevated SII might benefit from anti-inflammatory and/or anti-immunotherapy.

Acknowledgments Open access funding provided by Medical University of Vienna. The authors would like to thank Dr. Helmuth Haslacher for his assistance in the Material and Methods section (laboratory methods).

Author Contribution All authors meet all the following criteria: substantial contributions to the conception or design of the work, or the acquisition, analysis, or interpretation of data for the work; drafting the work or revising it critically for important intellectual content; final approval of the version to be published; and agreement to be accountable for all aspects of the work in ensuring that questions related to the accuracy or integrity of any part of the work are appropriately investigated and resolved.

\section{Compliance with Ethical Standards}

Conflict of Interest The authors declare no conflict of interest.

Open Access This article is distributed under the terms of the Creative Commons Attribution 4.0 International License (http:// creativecommons.org/licenses/by/4.0/), which permits unrestricted use, distribution, and reproduction in any medium, provided you give appropriate credit to the original author(s) and the source, provide a link to the Creative Commons license, and indicate if changes were made.

\section{References}

1. Jemal A, Bray F, Center MM, Ferlay J, Ward E, Forman D. Global cancer statistics. CA Cancer J Clin. 2011;61(2):69-90. doi:https:// doi.org/10.3322/caac.20107.

2. Ferlay J, Parkin DM, Steliarova-Foucher E. Estimates of cancer incidence and mortality in Europe in 2008. Eur J Cancer. 2010;46(4):765-81. doi:https://doi.org/10.1016/j.ejca.2009.12. 014.

3. Grivennikov SI, Greten FR, Karin M. Immunity, inflammation, and cancer. Cell. 2010;140(6):883-99. doi:https://doi.org/10.1016/j. cell.2010.01.025.

4. Hanahan D, Weinberg RA. Hallmarks of cancer: the next generation. Cell. 2011;144(5):646-74. doi:https://doi.org/10.1016/j.cell. 2011.02.013.

5. Mantovani A, Allavena P, Sica A, Balkwill F. Cancer-related inflammation. Nature. 2008;454(7203):436-44. doi:https://doi.org/ 10.1038 /nature 07205 . 
6. Proctor MJ, Morrison DS, Talwar D, Balmer SM, O'Reilly DS, Foulis AK et al. An inflammation-based prognostic score (mGPS) predicts cancer survival independent of tumour site: a Glasgow Inflammation Outcome Study. Br J Cancer. 2011;104(4):726-34. doi:https://doi.org/10.1038/sj.bjc.6606087.

7. Jomrich G, Hollenstein M, John M, Baierl A, Paireder M, Kristo I et al. The modified glasgow prognostic score is an independent prognostic indicator in neoadjuvantly treated adenocarcinoma of the esophagogastric junction. Oncotarget. 2018;9(6):6968-76. doi:https://doi.org/10.18632/oncotarget.24087.

8. Martinelli P, Carrillo-de Santa Pau E, Cox T, Sainz B, Jr, Dusetti N, Greenhalf W et al. GATA6 regulates EMT and tumour dissemination, and is a marker of response to adjuvant chemotherapy in pancreatic cancer. Gut. 2017;66(9):1665-76. doi:https://doi.org/10. 1136/gutjnl-2015-311256.

9. Pelosi E, Castelli G, Testa U. Pancreatic Cancer: Molecular Characterization, Clonal Evolution and Cancer Stem Cells. Biomedicines. 2017;5(4). https://doi.org/10.3390/ biomedicines 5040065 .

10. Fidler IJ, Poste G. The "seed and soil" hypothesis revisited. Lancet Oncol. 2008;9(8):808. doi:https://doi.org/10.1016/S1470-2045(08) 70201-8.

11. Perini MV, Montagnini AL, Jukemura J, Penteado S, Abdo EE, Patzina $\mathrm{R}$ et al. Clinical and pathologic prognostic factors for curative resection for pancreatic cancer. HPB (Oxford). 2008;10(5): 356-62. doi:https://doi.org/10.1080/13651820802140752.

12. Aziz MH, Sideras K, Aziz NA, Mauff K, Haen R, Roos D et al. The Systemic-Immune-Inflammation Index Independently Predicts Survival and Recurrence in Resectable Pancreatic Cancer and its Prognostic Value Depends on Bilirubin Levels: A Retrospective Multicenter Cohort Study. Ann Surg. 2018. doi:https://doi.org/10. 1097/SLA.0000000000002660.

13. Feng JF, Chen S, Yang X. Systemic immune-inflammation index (SII) is a useful prognostic indicator for patients with squamous cell carcinoma of the esophagus. Medicine (Baltimore). 2017;96(4): e5886. doi:https://doi.org/10.1097/MD.0000000000005886.

14. Geng Y, Shao Y, Zhu D, Zheng X, Zhou Q, Zhou W et al. Systemic Immune-Inflammation Index Predicts Prognosis of Patients with Esophageal Squamous Cell Carcinoma: A Propensity Scorematched Analysis. Sci Rep. 2016;6:39482. doi:https://doi.org/10. 1038/srep39482.

15. Hong X, Cui B, Wang M, Yang Z, Wang L, Xu Q. Systemic Immune-inflammation Index, Based on Platelet Counts and Neutrophil-Lymphocyte Ratio, Is Useful for Predicting Prognosis in Small Cell Lung Cancer. Tohoku J Exp Med. 2015;236(4):297304. doi:https://doi.org/10.1620/tjem.236.297.

16. Hu B, Yang XR, Xu Y, Sun YF, Sun C, Guo W et al. Systemic immune-inflammation index predicts prognosis of patients after curative resection for hepatocellular carcinoma. Clin Cancer Res. 2014;20(23):6212-22. doi:https://doi.org/10.1158/1078-0432. CCR-14-0442.

17. Tempero MA, Malafa MP, Al-Hawary M, Asbun H, Bain A, Behrman SW et al. Pancreatic Adenocarcinoma, Version 2.2017, NCCN Clinical Practice Guidelines in Oncology. J Natl Compr Canc Netw. 2017;15(8):1028-61. doi:https://doi.org/10.6004/ jnccn.2017.0131.

18. R Development Core Team. R: A language and environment for statistical computing. Vienna: R Foundation for Statistical Computing; 2018.

19. Robin X, Turck N, Hainard A, Tiberti N, Lisacek F, Sanchez JC et al. pROC: an open-source package for $\mathrm{R}$ and $\mathrm{S}+$ to analyze and compare ROC curves. BMC Bioinformatics. 2011;12:77. doi: https://doi.org/10.1186/1471-2105-12-77.

20. Therneau T. A Package for Survival Analysis in S. 2.38 ed2015.

21. López-Ratón M, Rodríguez-Álvarez MX, Cadarso-Suárez C, Gude-Sampedro F. OptimalCutpoints: An R Package for
Selecting Optimal Cutpoints in Diagnostic Tests. J Stat Soft. 2014;61(8). https://doi.org/10.18637/jss.v061.i08.

22. Youden WJ. Index for rating diagnostic tests. Cancer. 1950;3(1): $32-5$.

23. Powers DMW. Evaluation: From precision, recall and f-measure to roc., informedness, markedness \& correlation. Journal of Machine Learning Technologies. 2011;2(1):37-63.

24. Balkwill F, Mantovani A. Inflammation and cancer: back to Virchow? Lancet. 2001;357(9255):539-45. doi:https://doi.org/10. 1016/S0140-6736(00)04046-0.

25. Dvorak HF. Tumors: wounds that do not heal. Similarities between tumor stroma generation and wound healing. N Engl J Med. 1986;315(26):1650-9. doi:https://doi.org/10.1056/ NEJM198612253152606.

26. Guthrie GJ, Roxburgh CS, Farhan-Alanie OM, Horgan PG, McMillan DC. Comparison of the prognostic value of longitudinal measurements of systemic inflammation in patients undergoing curative resection of colorectal cancer. Br J Cancer. 2013;109(1): 24-8. doi:https://doi.org/10.1038/bjc.2013.330.

27. Jomrich G, Paireder M, Gleiss A, Kristo I, Harpain L, Schoppmann SF. Comparison of Inflammation-Based Prognostic Scores in a Cohort of Patients with Resectable Esophageal Cancer. Gastroenterol Res Pract. 2017;2017:1678584. doi:https://doi.org/ 10.1155/2017/1678584.

28. Pinato DJ, Shiner RJ, Seckl MJ, Stebbing J, Sharma R, Mauri FA. Prognostic performance of inflammation-based prognostic indices in primary operable non-small cell lung cancer. Br J Cancer. 2014;110(8):1930-5. doi:https://doi.org/10.1038/bjc.2014.145.

29. Tong YS, Tan J, Zhou XL, Song YQ, Song YJ. Systemic immuneinflammation index predicting chemoradiation resistance and poor outcome in patients with stage III non-small cell lung cancer. J Transl Med. 2017;15(1):221. doi:https://doi.org/10.1186/s12967017-1326-1.

30. Lolli C, Basso U, Derosa L, Scarpi E, Sava T, Santoni M et al. Systemic immune-inflammation index predicts the clinical outcome in patients with metastatic renal cell cancer treated with sunitinib. Oncotarget. 2016;7(34):54564-71. doi:https://doi.org/10.18632/ oncotarget.10515.

31. Lolli C, Caffo O, Scarpi E, Aieta M, Conteduca V, Maines F et al. Systemic Immune-Inflammation Index Predicts the Clinical Outcome in Patients with mCRPC Treated with Abiraterone. Front Pharmacol. 2016;7:376. doi:https://doi.org/10.3389/fphar. 2016.00376.

32. McMillan DC. Cancer and systemic inflammation: stage the tumour and stage the host. Br J Cancer. 2013;109(3):529. doi: https://doi.org/10.1038/bjc.2013.418.

33. Houghton AM, Rzymkiewicz DM, Ji H, Gregory AD, Egea EE, Metz HE et al. Neutrophil elastase-mediated degradation of IRS-1 accelerates lung tumor growth. Nat Med. 2010;16(2):219-23. doi: https://doi.org/10.1038/nm.2084.

34. De Larco JE, Wuertz BR, Furcht LT. The potential role of neutrophils in promoting the metastatic phenotype of tumors releasing interleukin-8. Clin Cancer Res. 2004;10(15):4895-900. doi: https://doi.org/10.1158/1078-0432.CCR-03-0760.

35. Chen HC, Lin HC, Liu CY, Wang CH, Hwang T, Huang TT et al. Neutrophil elastase induces IL-8 synthesis by lung epithelial cells via the mitogen-activated protein kinase pathway. J Biomed Sci. 2004;11(1):49-58. doi:https://doi.org/10.1159/000075288.

36. Teramukai S, Kitano T, Kishida Y, Kawahara M, Kubota K, Komuta $\mathrm{K}$ et al. Pretreatment neutrophil count as an independent prognostic factor in advanced non-small-cell lung cancer: an analysis of Japan Multinational Trial Organisation LC00-03. Eur J Cancer. 2009;45(11):1950-8. doi:https://doi.org/10.1016/j.ejca. 2009.01.023.

37. Labelle M, Begum S, Hynes RO. Direct signaling between platelets and cancer cells induces an epithelial-mesenchymal-like transition 
and promotes metastasis. Cancer Cell. 2011;20(5):576-90. doi: https://doi.org/10.1016/j.ccr.2011.09.009.

38. Walsh SR, Cook EJ, Goulder F, Justin TA, Keeling NJ. Neutrophillymphocyte ratio as a prognostic factor in colorectal cancer. J Surg Oncol. 2005;91(3):181-4. doi:https://doi.org/10.1002/jso.20329.

39. Sarraf KM, Belcher E, Raevsky E, Nicholson AG, Goldstraw P, Lim E. Neutrophil/lymphocyte ratio and its association with survival after complete resection in non-small cell lung cancer. J Thorac Cardiovasc Surg. 2009;137(2):425-8. doi:https://doi.org/10.1016/j. jtcvs.2008.05.046.

40. Kao SC, Pavlakis N, Harvie R, Vardy JL, Boyer MJ, van Zandwijk $\mathrm{N}$ et al. High blood neutrophil-to-lymphocyte ratio is an indicator of poor prognosis in malignant mesothelioma patients undergoing systemic therapy. Clin Cancer Res. 2010;16(23):5805-13. doi:https:// doi.org/10.1158/1078-0432.CCR-10-2245.

41. Cho H, Hur HW, Kim SW, Kim SH, Kim JH, Kim YT et al. Pretreatment neutrophil to lymphocyte ratio is elevated in epithelial ovarian cancer and predicts survival after treatment. Cancer Immunol Immunother. 2009;58(1):15-23. doi:https://doi.org/10. 1007/s00262-008-0516-3.

42. Smith RA, Bosonnet L, Raraty M, Sutton R, Neoptolemos JP, Campbell $\mathrm{F}$ et al. Preoperative platelet-lymphocyte ratio is an independent significant prognostic marker in resected pancreatic ductal adenocarcinoma. Am J Surg. 2009;197(4):466-72. doi: https://doi.org/10.1016/j.amjsurg.2007.12.057.

43. Chawla A, Huang TL, Ibrahim AM, Hardacre JM, Siegel C, Ammori JB. Pretherapy neutrophil to lymphocyte ratio and platelet to lymphocyte ratio do not predict survival in resectable pancreatic cancer. HPB (Oxford). 2018;20(5):398-404. doi:https://doi.org/10. 1016/j.hpb.2017.10.011.

44. Mowbray NG, Griffith D, Hammoda M, Shingler G, Kambal A, AlSarireh B. A meta-analysis of the utility of the neutrophil-tolymphocyte ratio in predicting survival after pancreatic cancer resection. HPB (Oxford). 2018;20(5):379-84. doi:https://doi.org/10. 1016/j.hpb.2017.12.009.

45. Haldar R, Ben-Eliyahu S. Reducing the risk of post-surgical cancer recurrence: a perioperative anti-inflammatory anti-stress approach. Future Oncol. 2018;14(11):1017-21. doi:https://doi.org/10.2217/ fon-2017-0635.

Publisher's Note Springer Nature remains neutral with regard to jurisdictional claims in published maps and institutional affiliations. 